\title{
Freqüência de ácaros em cães e gatos no município de Mossoró, Rio Grande do Norte
}

\author{
Mites frequency in dogs and cats at the city of Mossoro, Rio Grande do Norte, Brazil \\ Gilton Silva da Rocha ${ }^{1}$, Sílvia Maria Mendes Ahid ${ }^{2}$, Ana Carla Diógenes Suassuna Bezerra ${ }^{2}$, Kilder \\ Dantas Filgueira $^{3} \&$ Julianna Pereira da Silva Santos ${ }^{1}$
}

\begin{abstract}
RESUMO
Os ectoparasitos são causadores de dermatopatias nos animais domésticos, acometendo, freqüentemente, cães e gatos e podendo assumir um possível potencial zoonótico. Assim, o presente trabalho teve por objetivo descrever a ocorrência de ácaros, causadores de sarnas, em cães e gatos, no município de Mossoró, estado do Rio Grande do Norte. O estudo foi conduzido entre fevereiro de 2002 a janeiro de 2007, no qual foram coletadas amostras de 503 animais (entre cães e gatos), através de raspados cutâneos, individualmente identificados e diagnosticados no Laboratório de Parasitologia Animal da Universidade Federal Rural do Semi-Árido (UFERSA). A análise dos dados foi realizada por meio de frequêencia absoluta e relativa. Em relação aos cães, 77 (18,6\%) apresentaram alguma espécie de ácaro, com 70 (90,9\%) positivos para Demodex canis e 7 (9\%) acometidos por Sarcoptes scabiei var. canis, enquanto que, em gatos, os positivos representaram 26 (28,5\%) animais, com presença de $18(69,2 \%)$ felinos com Notoedres cati, 4 (15,3\%) positivos para S. scabiei e 4 (15,3\%) com Lynxacarus radovskyi. Os caninos e felinos estudados apresentaram ácaros distintos.
\end{abstract}

Descritores: dermatologia, ácaros, diagnóstico, ocorrência, carnívoros domésticos.

\begin{abstract}
Ectoparasites cause skin diseases in domestic animals, they often afflict dogs and cats, it may be a possible zoonotic potential. Thus, the present work aimed to describe the mites occurrence, that causes scabes, in dogs and cats, at the city of Mossoro, Rio Grande do Norte state. The study was conducted between february 2002 and january 2007, when there were collected samples from 503 animals (among dogs and cats) through cutaneous smears, individually identified and diagnosised at the Animal Parasitological Laboratory of the Rural Federal University of the semi-arid (UFERSA). The data analysis was performed by means of absolute and relative frequency. Among the dogs, 77 (18.6\%) presented some species of mite, from which $70(90.9 \%)$ were tested positive for Demodex canis and 7 (9\%) were tested positive for Sarcoptes scabiei var. canis, meanwhile, 26 cats $(28.5 \%)$ were tested positive, from which $18(69.2 \%)$ felines with Notoedres cati, $4(15.3 \%)$ tested positive for S. Scabiei and 4 (15.3\%) with Lynxacarus radovskyi. The canines and felines studied here presented distinctive mites.
\end{abstract}

Key words: dermatology, mites, diagnostic, occurrence, domestic carnivores. 


\section{INTRODUÇÃO}

Os distúrbios da pele representam cerca de 30 a $40 \%$ do atendimento clínico de carnívoros domésticos, independente da localização geográfica e do desenvolvimento sócio-econômico do país considerado [13], com as dermatites parasitárias assumindo um papel exponencial, devido à magnitude da ocorrência, sinais clínicos e potencial zoonótico existente [4].

Dentre as doenças cutâneas de origem parasitária, estão as acarioses, causadas por ácaros que ocorrem freqüentemente em cães e gatos. Entre esses, destacam-se as espécies Sarcoptes scabiei var canis, Notoedres cati, Otodectes cynotis e Demodex canis [11], além do Lynxacarus radovskyi, que hoje se apresenta como um problema crescente na população de felinos domésticos [1].

O ectoparasitismo por ácaros, em carnívoros domésticos, tem sido relatado em diversas regiões do Brasil, com estudos na região Sudeste [1,5,12], no Sul $[4,11]$ e alguns estados do Nordeste $[2,6,8,10,14$, 17].

Os ácaros mostram variações morfológicas e biológicas estritamente relacionadas com sua adaptação ao meio em que vivem. Logo, é necessária a adoção de medidas relacionadas ao controle, as quais são precedidas de estudos sobre os ectoparasitos presentes na região, a fim de direcionar as ações de prevenção, otimizando os recursos e, principalmente, diminuindo o impacto ambiental causado pelo controle químico indiscriminado [17].

Tendo em vista a importância desses ectoparasitos na prática da clínica veterinária de pequenos animais, o objetivo deste trabalho foi descrever a ocorrência de ácaros em cães e gatos, no município de Mossoró.

\section{MATERIAIS E MÉTODOS}

O estudo foi conduzido no período de fevereiro de 2002 a janeiro de 2007, no município de Mossoró, estado do Rio Grande do Norte. As amostras foram coletadas em animais que apresentavam lesões dermatológicas, provenientes da rotina clínica do Hospital Veterinário da Universidade Federal Rural do Semi-Árido (UFERSA), e de visitas semanais ao Centro de Controle de Zoonoses (CCZ) de Mossoró. As amostras foram enviadas para análise no Laboratório de Parasitologia Animal da UFERSA. O material oriundo de cada animal foi acondicionado entre lâminas de microscopia, em que instilou-se de duas a três gotas de hidróxido de potássio a $10 \%$, aguardando-se 10 minutos. Em seguida, foi sobreposta uma lamínula e examinou-se o material em microscópio óptico (10X e 20X) e estereomicroscópio (quando necessário), com posterior identificação [9], de acordo com as características morfológicas do parasito. Os dados obtidos foram distribuídos em frequiência absoluta e percentual.

\section{RESULTADOS}

Foram examinadas 503 amostras, sendo 412 (82\%) de cães e 91 (18\%) de gatos. Em relação aos cães, 77 (18,6\%) apresentaram alguma espécie de ácaro, com $70(90,9 \%)$ positivos para Demodex canis e 7 (9\%) Sarcoptes scabiei var. canis.

As raças que apresentaram positividade foram: Pit Bull (40,5\%), Husky Siberiano (100\%), Doberman (8,3\%), Dogue Alemão (50\%), Rotweiller (30,7\%), Pinscher (17,6\%), Pastor Alemão (20,7\%) e Fila Brasileiro (40\%), entretanto, nos cães Sem Raça Definida (SRD), foi verificado que $12,2 \%$ apresentaram $D$. canis e 2,3\% S. scabiei var. canis (Tabela 1).

Em gatos, os positivos representaram 26 $(28,5 \%)$ dos 91 atendidos, com presença de $18(69,2 \%)$ felinos com Notoedres cati, 4 (15,3\%) com S. scabiei e $4(15,3 \%)$ positivos para o Lynxacarus radovskyi. Nos siameses, observou-se o S. scabiei em 1 (50\%) animal e o $N$. cati também em apenas $1(50 \%)$ felino. Naqueles da raça persa, foi verificado $1(25 \%)$ animal com $N$. cati e $3(75 \%)$ com L. radovskyi. Nos SRD ocorreu a presença de $N$. cati em 16 (80\%) animais, S. scabiei em 3 (15\%) felinos e L. radovskyi em 1 (5\%) gato (Tabela 1).

\section{DISCUSSÃO}

No município de Mossoró, o número de cães (412) examinados foi superior ao de gatos (91). Em estudos de prevalência, realizados no Rio Grande do Sul, o número de cães estudados $(95,6 \%)$ também foi superior ao de gatos $(4,4 \%)$ [11], demonstrando que, a frequiência de atendimentos clínicos em caninos, geralmente, é maior.

Em relação aos cães, $70(90,9 \%)$ animais apresentaram positividade para Demodex canis e 7 (9\%) Sarcoptes scabiei var. canis, concordando com estudos no estado de Goiás, que entre 31 cães atendidos foi possível verificar afecções de origem parasitária em 
Tabela 1. Ácaros identificados, por espécie, em raspados cutâneos de cães e gatos, durante o período de fevereiro de 2002 a janeiro de 2007, no município de Mossoró, Estado do Rio Grande do Norte.

\begin{tabular}{|c|c|c|c|c|c|}
\hline Espécie & Raças & $\begin{array}{c}\text { D. canis } \\
\text { N }(\%)\end{array}$ & $\begin{array}{c}\text { S. scabiei } \\
\mathrm{N}(\%)\end{array}$ & $\begin{array}{l}\text { N. cati } \\
\mathrm{N}(\%)\end{array}$ & $\begin{array}{l}\text { L. radovskyi } \\
\mathbf{N}(\%)\end{array}$ \\
\hline \multirow[t]{3}{*}{ Felina } & Siamês & $0(0,0)$ & $1(50,0)$ & $1(50,0)$ & $0(0,0)$ \\
\hline & Persa & $0(0,0)$ & $0(0,0)$ & $1(25,0)$ & $3(75,0)$ \\
\hline & SPRD & $0(0,0)$ & $3(15,0)$ & $16(80,0)$ & $1(5,0)$ \\
\hline \multirow[t]{9}{*}{ Canina } & Pit Bull & $28(40,5)$ & $0(0,0)$ & -- & -- \\
\hline & Husky & $1(50,0)$ & $1(50,0)$ & -- & -- \\
\hline & Doberman & $1(8,3)$ & $0(0,0)$ & -- & -- \\
\hline & Dogue Alemão & $1(50,0)$ & $0(0,0)$ & -- & -- \\
\hline & Rottweiler & $4(30,7)$ & $0(0,0)$ & -- & -- \\
\hline & Pinscher & $3(17,6)$ & $0(0,0)$ & -- & -- \\
\hline & Pastor Alemão & $4(16,6)$ & $1(4,1)$ & -- & -- \\
\hline & Fila Brasileiro & $2(40,0)$ & $0(0,0)$ & -- & -- \\
\hline & SPRD & $26(12,2)$ & $5(2,35)$ & -- & -- \\
\hline
\end{tabular}

$\boldsymbol{N}=$ Número absoluto; $\mathbf{S P R D}=$ Sem padrão de raça definido.

45,2\% dos casos, com a demodicidose em cães púberes destacando-se como a mais amplamente diagnosticada (78,6\%), seguida de S. scabiei var. canis (14,3\%) e tungíase $(7,1 \%)$ [13]. Entretanto, discordando desses dados, estudos realizados em Pernambuco, com cães, demonstraram que o $D$. canis foi observado apenas com uma prevalência de $0,5 \%$ a 2,7\% [17]. Já no estado de Santa Catarina, encontrou-se associação de ácaros em um canino, apresentando D. canis e S. scabiei var. canis concomitantemente [4]. Entre as acarioses, independentemente do tipo de pelame, a demodicidose é uma dermatopatia de animais com precisa definição racial $[16,3]$.

Em gatos, no município de Mossoró, os positivos representaram $26(28,5 \%)$ dos 91 atendidos, com presença de 18 (69,2\%) felinos com Notoedres cati, em concordância com estudos realizados com felinos dermatopatas oriundos do atendimento clínico de um hospital veterinário universitário no estado de Goiás, onde diagnosticaram a sarna notoédrica em 18,2\% dos casos [13]. Além de que, no Rio Grande do Sul, o N. cati $(33,3 \%)$ foi o ácaro mais freqüente [11].
Já o Lynxacarus radovskyi foi diagnosticado em $4(15,3 \%)$ felinos, concordando com outros relatos no estado do Rio Grande do Norte, que, de 20 animais examinados, 3 (15\%) mostraram-se positivos para $L$. radovskyi [2]. Na região metropolitana do Recife, dos 335 felinos estudados, $254(75,8 \%)$ apresentavam-se infestados por $L$. radovskyi $(75,82 \%)$, sem diferenciação de cor de pelagem para o parasitismo, porém há quem defenda que as infestações são maiores naqueles de pêlo escuro [15]. Na região metropolitana do Rio de Janeiro, estudos realizados no decorrer de um ano verificaram que, de 613 felinos examinados, 42 (7\%) estavam com linxacariose, com a frequiência nos machos, aproximadamente, três vezes mais alta do que nas fêmeas, com todos os felinos apresentando pêlo longo [7].

\section{CONCLUSÕES}

Os cães e gatos do município de Mossoró são acometidos por ácaros, tendo como principais o Demodex canis e Sarcoptes scabiei var. canis nos caninos e Notoedres cati, S. scabiei e Lynxacarus radovskyi em felinos. 


\section{REFERÊNCIAS}

1 Accetta E.M.T., Accetta J.L., Ferreira J.C.A. \& Rocha J.S.M. 2007. Relatos de 39 casos de linxacariose felina na região dos lagos do Rio de Janeiro. In: Resumos do XXVII Congresso Paulista de Clínicos de Pequenos Animais (São Paulo, Conpavepa). pp.7-9.

2 Ahid S.M.M.,Alves N.D., Suassuna A.C.D., Filgueira K.D., Saraiva M.V.A. \& Rocha G.S. 2005. Ocorrência de Lynxacarus radovskyi (Tenório, 1974) em gatos no município de Mossoró- RN. Nosso Clínico. 48: 56-60.

3 Batista L.M. \& Scutato F.H. 2008. Eficácia da impressão em fita adesiva no diagnóstico da demodiciose canina. Nosso Clínico. 61: 12-14.

4 Bellato V., Sartor A.A., Souza A.P. \& Ramos B.C. 2003. Ectoparasitos em caninos do município de Lages, Santa Catarina, Brasil. Revista Brasileira de Parasitologia Veterinária. 12: 95-98.

5 Costa J.O., Guimarães M.P. \& Lima W.S. 1990. Freqüência de endo e ectoparasitos de cães capturados nas ruas de Vitória-ES, Brasil. Arquivo Brasileiro de Medicina Veterinária. 42: 451-452.

6 Ferreira D.R.A., Cunha E.M.N., Faustino A.G. \& Alves L.C. 2005. Lynxacarus radovskyi (Tenório, 1974) em felinos na cidade de João Pessoa- Paraíba: relato de caso. In: Resumo do XXVI Congresso Brasileiro de Clínicos Veterinários de Pequenos Animais (Salvador, ANCLIVEPA Brasil). pp.166-167.

7 Figueiredo F.B., Schubach T.M.P., Pereira S.A., Okamoto T., Santos I.B., Leme L.R.P. \& Faccini J.L.H. 2004. Relato de mais 42 casos de linxacariose na região metropolitana da cidade do Rio de Janeiro. Revista Brasileira de Parasitologia. 13: $41-43$.

8 Gondin L.F.P., Conceição L.G., Franco S.R.V.S. \& Kohayagama A. 1994. Lynxacarus radovskyi em gatos: descrição de um caso. In: Anais do XXVIII Congresso Brasileiro de Medicina Veterinária (Recife, Sociedade Pernambucana de Medicina Veterinária). p.672.

9 Guimarães J.H., Tucci E.C. \& Barros-Battesti D.M. 2001. Ectoparasitos de Importância Veterinária. São Paulo: FAPESP, $218 \mathrm{p}$.

10 Matos M.S., Souza R.M., Matos P.F., Costa J.A. \& Santos L.M.M. 1982. Frequency of mange mites on dogs in Salvador, Bahia. Arquivo Escola Médica Veterinária da Universidade Federal da Bahia. 7: 91-96.

11 Neuwald E.B., Ribeiro V.L.S., Seibert M. \& Torres J.R. 2004. Prevalência das acarioses de cães e gatos diagnosticados no laboratório de entomologia da FAVET/UFGS de 2000 a 2003. In: Resumo do XXV Congresso Brasileiro de Clínicos Veterinários de Pequenos Animais. (Gramado: ANCLIVEPA Brasil). p.40.

12 Rodrigues A.F.S.F., Daemon E. \& D’agosto M. 2001. Investigação sobre alguns ectoparasitos em cães de rua no município de Juiz de Fora, Minas Gerais. Revista Brasileira de Parasitologia Veterinária. 10: 13-19.

13 Romani A.F., Freitas K.C., Rabelo R.E., Sant'ana F.J.F., Fernandes J.J.R., Silva C.C., Lobo M.B. \& Amaral A.V.C. 2005. Ocorrência de dermatopatias em pequenos animais na cidade de Jataí-Go: resultados parciais. In: Resumo do XXVI Congresso Brasileiro de Clínicos Veterinários de Pequenos Animais. (Salvador: ANCLIVEPA Brasil). pp.133-134.

14 Romeiro E.T., Faustino M.A.G., Alves L.C., Soares Y.M.V. \& Matoso U.N.V. 2007. Aspectos clínicos da infestação por Lynxacarus radovskyi em gatos procedentes da região metropolitana do Recife. Clínica Veterinária. 69: 108-113.

15 Romeiro E.T., Alves L.C., Soares Y.M.V., Matoso U.N.V. \& Faustino M.A.G. 2007. Infestação por Lynxacarus radovskyi (Tenorio, 1974) em gatos domésticos procedentes da região metropolitana do Recife, Pernambuco, Brasil. Revista Brasileira de Parasitologia. 16: 159-162.

16 Scott D.W., Miller Jr W.H. \& Griffin C.E. 1996. Dermatologia de Pequenos Animais. 5.ed. Rio de Janeiro: Interlivros, $1142 \mathrm{p}$.

17 Torres F.D., Figueiredo L.A. \& Faustino M.A.D.A.G. 2004. Ectoparasitos de cães provenientes de alguns municípios da região metropolitana do Recife, Pernambuco, Brasil. Revista Brasileira de Parasitologia Veterinária. 13: 151-154.

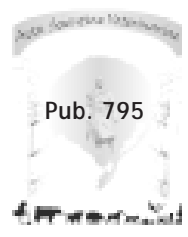

